\title{
Children's attention to online adverts is related to low-level saliency factors and individual level of gaze control
}

\author{
Nils Holmberg \\ Lund University
}

\author{
Kenneth Holmqvist \\ Lund University
}

\author{
Helena Sandberg \\ Lund University
}

\begin{abstract}
Twenty-six children in 3rd grade were observed while surfing freely on their favourite websites. Eye movement data were recorded, as well as synchronized screen recordings. Each online advert was analyzed in order to quantify low-level saliency features, such as motion, luminance and edge density. The eye movement data were used to register if the children had attended to the online adverts. A mixed-effects multiple regression analysis was performed in order to test the relationship between visual attention on adverts and advert saliency features. The regression model also included individual level of gaze control and level of internet use as predictors. The results show that all measures of visual saliency had effects on children's visual attention, but these effects were modulated by children's individual level of gaze control.
\end{abstract}

Keywords: children, online advertising, visual attention, visual saliency, internet use

\section{Introduction}

Current media research indicates that Swedish 9year-old children spend about one hour online every day (Medierådet, 2013; Nordicom, 2013). Although this average statistic is likely to be representative of this particular population, research also shows that there is quite a lot of variability around this central tendency. Thus, there is a fairly large group of heavy internet users among Swedish 9-year-olds, but also large groups of children that have very little contact with the internet (Medierådet, 2013; Holmberg, Sandberg, \& Holmqvist, 2014). In this research, heavy internet usage is defined as more than 3 hours of daily internet use. To some extent these differences in internet use can be explained by demographic factors such as gender and social class (e.g. boys are usually overrepresented among heavy internet users) (Medierådet, 2013). Looking at the media development over the last couple of years, there is a clear trend that daily internet use among children is increasing in younger age groups (Medierådet, 2013; Nordicom, 2013). Typical online activities among 9-year-olds are playing games and watching video clips (Carlsson, 2012). As children grow older, online gaming activities are gradually replaced by activities on social networking websites (Carlsson, 2012). Similar internet usage patterns are found in all Nordic countries.

In parallel to this general increase in children's online activity, the internet is also growing rapidly as a commercial advertising channel. In Sweden, between
2005 and 2010, investments in internet advertising increased more than any other type of advertising, both in absolute and relative measures (Sundin, 2013). The popularity of the internet as an advertising channel can be explained to some extent by the new and unique affordances that this medium provides in terms of advertising formats and strategies. An important new advertising technology on the internet has been labeled "behavioral targeting", and allows advertisers to track users' online behavior in order to infer their individual characteristics and interests (Singer \& Singer, 2011). Based on such user profiles, advertisers are able to serve up more relevant and better targeted adverts to website users (Goldfarb \& Tucker, 2011). On the other hand, the digital medium also allows for manipulations of basic visual features of online adverts in order to attract users' visual attention. Flashing banners, animations and pop-up ads are examples of how salient visual features such as motion and abrupt onset are incorporated into internet adverts to increase attentional capture (Ludwig, Ranson, \& Gilchrist, 2008; Parkhurst \& Niebur, 2004).

Since children's internet use is rising at the same time as this media environment is becoming more commercialized, we think it is important to measure the level of visual saliency in adverts found on children's websites, and investigate how these visual features affect children's exposure to adverts during ordinary internet use. At 9 years of age, children are undergoing rapid development in terms of voluntary attention, executive control, and decision-making, which poten- 
Journal of Eye Movement Research $8(2): 2,1-10$

tially makes them more sensitive to salient adverts than adults (Bucci \& Seassau, 2012; Davidson, Amso, Anderson, \& Diamond, 2006). Interview studies with children concerning their experiences of online advertising suggest that children tend to find adverts irritating and intrusive, and they express a feeling of being "followed" by adverts while surfing across their favourite websites (Martinez, Sandberg, \& Jarlbro, 2013). While internet usage statistics can provide a general outline of children's online activities, interviews studies can capture childrens experiences of online adverts. However, none of these methods are suited to give detailed information about children's actual visual exposure to online advertising as it occurs in real-time. In this exploratory study, we use eye-tracking to measure childrens visual attention, and we formulate several hypotheses concerning how individual factors and advert properties impact children's exposure to online adverts.

\section{Advert saliency and visual attention}

Visual saliency refers to the fact that certain visual features can make objects "pop out" from the surround and impinge on viewers' visual attention. Many such saliency features (e.g. motion, contrast, color, and luminance) can be extracted from a visual scene by using computer algorithms to produce so-called "saliency maps" (Itti \& Koch, 2000). Eye-tracking studies have shown that such saliency maps to some extent can predict where viewers will fixate (Foulsham \& Underwood, 2008). Although visual features in internet adverts can be analyzed in order to quantify their visual saliency, the media research in this area is scarce. Furthermore, there is little research concerning child audiences' actual visual exposure to internet adverts. It is possible to theorize that children's visual exposure to online adverts is low, based on the assumption that children engage in task-oriented behavior while surfing on the internet, which enables them to ignore visual stimuli from task-irrelevant adverts (Malcolm \& Henderson, 2010). Another possibility is that children actually do look at online adverts, but only when the advert content is relevant to their personal interests, and not depending on basic visual features. Currently, there is a fairly strong consensus that, while low-level visual features such as abrupt onset can account for some portion of people's eye movement behavior, relevant content is more powerful in explaining visual attention allocation (Tatler, Hayhoe, Land, \& Ballard, 2011; Foulsham \& Underwood, 2008). In order to further this debate, we measure visual saliency in internet adverts and hypothesize how these visual features affect children's visual attention (H1).

- H1: Higher levels of saliency will be associated with more visual attention to online ads.
Holmberg, N., Holmqvist, K., \& Sandberg, H. (2015) Online Adverts Affect Children's Attention

\section{Children's gaze control}

When visual attention is driven by salient features, it is referred to as exogeneous or stimulusdriven attention. Several studies have shown that the capacity to inhibit stimulus-driven, reflexive eye movements undergoes significant development throughout childhood, which is linked to ongoing development of the frontal lobe (Klein \& Foerster, 2001; Eenshuistra, Ridderinkhof, \& Molen, 2004). This research on stimulus-driven attention is relevant when investigating children's exposure to online advertising for two reasons: 1) younger children as a group could be more sensitive to visual saliency in online ads than adolescents and adults, and 2) younger children's individual level of gaze control could vary substantially due to rapid physiological and cognitive development, thereby predisposing some children for advertising exposure. In this study we operationalized the concept of gaze control by using an anti-saccade task to determine children's individual level of oculomotor control. This paradigm directly measures participants' voluntary control of their eye movements, and has been shown to correlate with cognitive functions such as executive control, working memory capacity and visual distractibility (Kramer, Gonzalez de Sather, \& Cassavaugh, 2005; Munoz \& Everling, 2004; Hutton \& Ettinger, 2006; Zanelli et al., 2005). To our knowledge, there have been no attempts to measure children's visual distractibility in relation to their advertising exposure. In the present study, we measure the gaze control of 9-year-old children, and hypothesize how their individual ability for gaze control affects their advertising exposure (H2).

- H2: Lower levels of gaze control will be associated with more visual attention to online ads.

\section{Internet use and advertising exposure}

Demographic factors can predict some aspects of children's internet use. Survey data from current media research in Sweden show that children in the tween age group (9-12 years) spend about 1-2 hours online each day. Boys and younger children are more likely to use the internet for playing online games, while girls and older children are more likely to spend their online time on social networking websites (Carlsson, 2012). Boys are also more likely to belong to the category of heavy internet users, that have 2-3 times higher internet usage compared to their peer average (Carlsson, 2012). However, these media usage surveys do not reveal if there is any relationship between childrens internet usage patterns and their online advertising exposure. While it is likely that the more time children spend online, the more they become subject to advertising messages, it is also necessary to take into account the type of web pages that different groups of children prefer to visit. E.g. websites containing online games 
Journal of Eye Movement Research $8(2): 2,1-10$

could display more advert content than social networking sites. These arguments suggest that 9-year-old boys could have a higher potential for advertising exposure than girls in the same age, which we hypothesize in this study (H3).

Previous research have also investigated adolescents' online gaze behavior, and found large individual differences between potential exposure (the number of adverts on web pages) and actual exposure (visual attention to adverts) (Gidlöf, Holmberg, \& Sandberg, 2012; Sandberg, Gidlöf, \& Holmberg, 2011). A possible explanation for these observed differences is that some individuals have more experience of using the internet for task-oriented purposes, and therefore have developed skills in avoiding online advertising. We know from other domains of research that gaze behavior differ significantly between experts and novices (Jarodzka, Scheiter, Gerjets, \& van Gog, 2010). Using weekly internet usage time as an indicator of internet literacy, we hypothesize that this measure impacts childrens visual attention to online ads (H4).

- H3: Male gender in children will be associated with more visual attention to online ads.

- H4: Less internet use will be associated with more visual attention to online ads.

In order to test the aforementioned hypotheses, we designed a quasi-experiment in which 9-year-old children were allowed to surf freely on their favourite websites. During these free web surfing session we recorded the children's eye movements as an indicator of their visual attention. We also recorded the dynamic web page stimuli that the children interacted with as real-time screen recordings. These screen recordings allowed us to extract the level of visual saliency in authentic online adverts and measure the effects of several saliency features on children's visual attention. In order to factor in the children's individual level of gaze control, an anti-saccade task was performed on all participants in the study.

\section{Methods}

Given the young age of the participants in this study, we assumed that undertaking the study in a familiar school environment would elicit the most natural web surfing behavior in the children. In addition, the eyetracking apparatus selected for this study was the least invasive. Taken together, these features increase the ecological validity of the current study design, compared to performing the study in a more controlled laboratory environment.

\section{Participants and apparatus}

An elementary school in the south of Sweden was selected for the study. The main selection criterion
Holmberg, N., Holmqvist, K., \& Sandberg, H. (2015) Online Adverts Affect Children's Attention

was that the school should be representative of the population in terms of demographics. 40 children in two Swedish 3rd grade classes were given forms for parental consent. 26 children were given parental consent and opted to partake in the study. Before partaking in the study, all children were given an internet usage questionnaire. Ages ranged from 9 years $(n=23)$ to 10 years $(n=3)$. The children had mixed ethnic backgrounds, but were all fluent in spoken Swedish. The gender distribution was fairly equal between girls $(n=14)$ and boys $(n=12)$. All children had normal or corrected-to-normal vision. One participant had to be excluded due to technical problems during the web browsing session.

The recording equipment consisted of a SMI RED 250 laptop system that was used for web surfing and eye movement recordings respectively (a singlecomputer setup). The laptop (Intel Core i7 $2.67 \mathrm{GHz}$ CPU, 2.98 GB RAM) was connected to the internet through a wireless 3G USB modem with a bandwidth of approximately $1 \mathrm{Mb} / \mathrm{s}$ (Huawei Mobile Broadband). The web page stimuli were presented on a $1680 \times 1050$ LCD monitor with a SMI remote eye-tracking device attached to the lower side. The children interacted with the web pages through an optic mouse and a USB keyboard, both of which were connected to the recording / stimulus presentation laptop. Audio playback was handled with desktop speakers connected with $15 \mathrm{~mm}$ TRS plug. Eye-tracking data were recorded at $250 \mathrm{~Hz}$ with SMI iViewX 2.7 software during all tasks, and concurrent screen recordings from the stimulus monitor were captured at $10 \mathrm{~Hz}$. All calibrations, validations, stimulus presentations, and screen recordings were handled using SMI ExperimentCenter 3.1 software.

\section{Study design and materials}

Before the internet surfing session, an anti-saccade test was presented on the stimulus monitor in order to test each participants' individual level of gaze control. Each trial in the anti-saccade test consisted of two stimulus events. First, a fixation cross was presented in the center of the screen with a duration of 1500-2000 ms. Second, the fixation cross was replaced by a peripheral target, presented ca $10 \mathrm{deg}$ off-center on the screen. As the target appeared, participants were instructed to saccade in the opposite direction. Targets were randomly presented horizontally (left or right of the fixation cross) or vertically (top or bottom), with a duration of $1000 \mathrm{~ms}$. After target offset, a blank screen was presented with a duration of $500 \mathrm{~ms}$. The antisaccade test consisted of 32 such trials. The stimulus parameters of the anti-saccade test were chosen in accordance with recently suggested standards for this paradigm (Antoniades et al., 2013). To reduce eye fatigue, all stimulus slides in the anti-saccade test were constructed with black background and white fixation 
Journal of Eye Movement Research $8(2): 2,1-10$

cross and targets.

After the anti-saccade pre-test, the children continued with free web surfing sessions. These sessions were implemented as follows: 1) A Microsoft Internet Explorer 8 window launched in full screen mode, and loaded a local HTML file containing a list of links to 47 websites; 2) The participants could select any link they preferred from the list and browse freely on the corresponding target website; 3 ) The participants could return to the initial link list at any time by hitting the browser's "home button", and select another website to browse. In addition, participants could search or enter any URL in the browser window, in case they wanted to browse websites not present in the link list. The link list was based on results from the internet usage survey, in which the children were encouraged to list all the websites they typically visited when going online. We assumed that this approach would facilitate natural web surfing while minimizing browser interaction difficulties (i.e. typing in URLs on the keyboard). The free surfing sessions were limited to 7 minutes. This time period was estimated to elicit a sufficient sample of advert exposures to gauge the effect of the selected saliency features on visual attention.

\section{Procedure}

Before each data recording, a general calibration protocol was followed, in order to calibrate the eyetracking system. An animated 2-point calibration procedure was displayed on the stimulus monitor, in which the participants were instructed to gaze directly at each calibration point until it changed position. Directly after the calibration, a 4-point validation was recorded to measure the deviation between gaze positions and validation targets. Calibrations were repeated until the deviation was below $1 \mathrm{deg}$ of visual angle (corresponding to ca $10 \mathrm{~mm}$ on the stimulus screen). The accuracy level and calibration protocol were designed to minimize the children's cognitive load before each data recording.

When the participant was sitting comfortably in front of the stimulus monitor, the calibration protocol described in the previous section was carried out, and then the anti-saccade test began. Verbal instructions were given to the children to think of the anti-saccade test as a game, in which the objective was to first keep the gaze directly at the fixation cross, and then avoid looking at the target dot presented peripherally, and instead look in the opposite direction. When we had ensured that the participants had understood the task, 4 training trials were initiated. After that, 32 actual trials were presented in 2 blocks of 16 trials each. Between the blocks there was a short intermission in order to debrief the participants.

After the anti-saccade test was completed, the participants proceeded directly to the free web surfing task. A 2-point re-calibration was made according to
Holmberg, N., Holmqvist, K., \& Sandberg, H. (2015) Online Adverts Affect Children's Attention

the general calibration protocol. Before the web surfing session started, the participants were instructed that a list of links to websites would appear in an ordinary browser window, and that the only task was to explore the list and navigate to any site they preferred to visit. The participants were also informed that the surfing session would be terminated automatically after a couple of minutes. The exact number of minutes was not mentioned in order not to confuse or stress the partcipants.

\section{Data analysis}

The overall quality of the eye-tracking data was calculated as the average deviation between the calibrated point of regard (POR) and 4 validation points. The average horizontal and vertical deviation was 0.75 deg and 0.92 deg respectively. The amount of missing samples (including blinks) in the anti-saccade data was $12.6 \%$. These quality measures were only calculated for the anti-saccade dataset, but they should generalize to the surfing dataset as well, since the exact same calibration procedure was applied in both cases. No participants had to be excluded due to poor data quality.

Eye movement data from the anti-saccade test were analyzed by using the Engbert and Kliegel algorithm in order to detect the first saccade in each trial (Engbert \& Kliegl, 2003). A minimum saccade duration of 32 ms was provided as a parameter for the detection algorithm. The first saccades were then analyzed for latency, peak velocity and direction relative to target position using a second algorithm (Ahlström et al., 2013). Saccade latency was calculated using a minimum latency parameter of $0.08 \mathrm{~ms}$, peak velocity was calculated using a maximum saccade velocity parameter of $1000 \mathrm{deg} / \mathrm{s}$. Anti-saccades were categorized binomially as correct if they were terminated within a $45 \mathrm{deg}$ angle in the opposite direction of the target location. Only the total proportion of correct anti-saccades for each participant was used for further analysis, as this construct was considered to be the most valid measure of gaze control.

Saliency analysis of the online adverts was achieved by using the screen-recordings obtained from the children's free surfing sessions. Each advert in this video data was defined in terms of its spatial and temporal location, and by using the OpenCV 2.3.1 software (Bradski, 2000), the regions corresponding to adverts were processed to extract three basic saliency measures. The saliency features extracted in this study was the amount of motion, luminance, and edges (Parkhurst \& Niebur, 2004). Motion was quantified by measuring the absolute difference in pixels between pairs of successive frames. Speed of motion was not considered in this study. Luminance was calculated by thresholding each frame and measuring the remaining luminance pixels. Edges were measured in each frame by using the OpenCV Canny function, returning 
Journal of Eye Movement Research $8(2): 2,1-10$
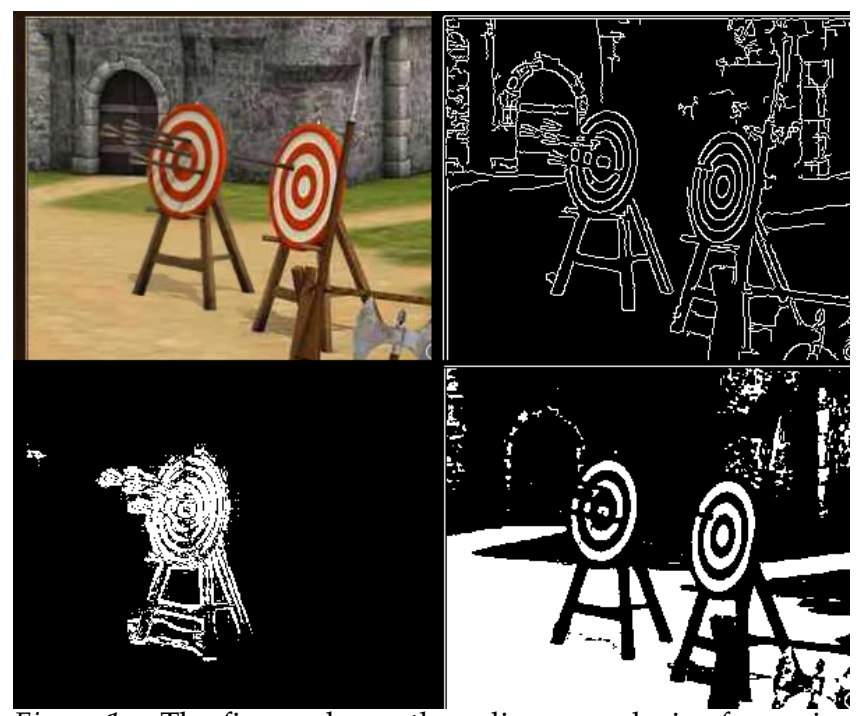

Figure 1. The figure shows the saliency analysis of one single frame from an animated advert: original advert (top-left), edge pixels (top-right), motion pixels (bottom-left), and luminance pixels (bottom-right). The number of white pixels in each sub-image constitutes the respective values for the saliency measures on this frame.

the number of edge pixels. These measures were also calculated for the entire frame in which the ad appeared, in order to calculate the relative saliency values for each advert (Figure 1).

Participants' eye movements during the free web surfing session were analyzed using the same screen recording videos that was used for the advert saliency analysis. These videos contained a circle indicating the subjects' gaze position that had been superimposed on the web page stimuli. By using the OpenCV MatchTemplate function, this circle was used to extract the pixel coordinates of the gaze point on the stimulus monitor. These pixel coordinates were then used to determine if the gaze point was within the rectangle of an advert on each video frame or sample. This samplebased variable was then aggregated to the advert level, which allowed for the construction of a binomial dependent variable that indicated if an advert had been looked at or not.

The video-based analysis employed in this study prohibited utilization of other typical gaze measures, such as dwell time and fixation durations on adverts. However, the reason for using the eye movement data as recorded in the screen recordings, was that these videos contained a time-locked representation of the participants' gaze position relative to dynamic visual events in the web page stimuli. In addition, we reasoned that a dependent measure that registered if an advert was looked at, rather than how much adverts were attended, would be more sensitive to the low-
Holmberg, N., Holmqvist, K., \& Sandberg, H. (2015) Online Adverts Affect Children's Attention

level saliency features investigated in this study.

\section{Results}

\section{Children's internet usage}

The internet usage survey generated 26 responses. The results from the survey show that most of the children in this study use their own computer $(50 \%)$ or a parent's computer $(69 \%)$ when accessing the internet at home. None of the children stated that they did not use the internet at all during an average week. The most common activity when spending time online was playing online games (96\%), closely followed by watching video clips (88\%) and listening to music (81\%). On average, the children spent ca 60 minutes on web browsing per day. This is very close to the current national average, which indicates that the sample in this study is representative of the larger population (Council, 2010). There are also some interesting differences in average internet usage time depending on gender, with boys spending more than double daily time on the internet compared to girls (92 vs 36 minutes). However, these results on internet usage times include two male outlier cases that had great impact on the averages. A two-tailed heteroscedastic t-test revealed no significant difference in internet usage time between genders $(\mathrm{p}=0.10)$

\section{Children's gaze control}

The proportion of valid anti-saccade trials that was kept for analysis was fairly high (ca 82\%). However, the proportion of correct responses was low, indicating that the children had difficulties inhibiting saccades towards the distractor, and saccading in the opposite direction at target onset. The proportion of correct saccades was $21 \%$, which is consistent with other research findings on saccadic eye movements in children (Bucci \& Seassau, 2012; Fukushima, Hatta, \& Fukushima, 2000). In the correct anti-saccade trials, saccade latencies were $474 \mathrm{~ms}$ on average, and $377 \mathrm{~ms}$ in incorrect trials. There were no differences in saccade latency depending on gender.

The overall results on anti-saccade accuracy and anti-saccade latency were expected, but a more interesting result of the anti-saccade test appeared when we looked at correct responses by gender. These results revealed that, in the current sample, boys seem to have a significantly higher success rate than girls. The average proportion of correct responses in girls was 0.16 , and 0.27 in boys (see Figure 2). This difference was significant $(\mathrm{p} \leq 0.05)$. This result is somewhat surprising since girls are generally assumed to have an earlier cognitive development at this age, which would supposedly facilitate correct responses in the anti-saccade test compared to boys (Patterson, 2008). Because of this difference, we decided to add an interaction between 
Journal of Eye Movement Research $8(2): 2,1-10$

Average correct anti-saccades by gender

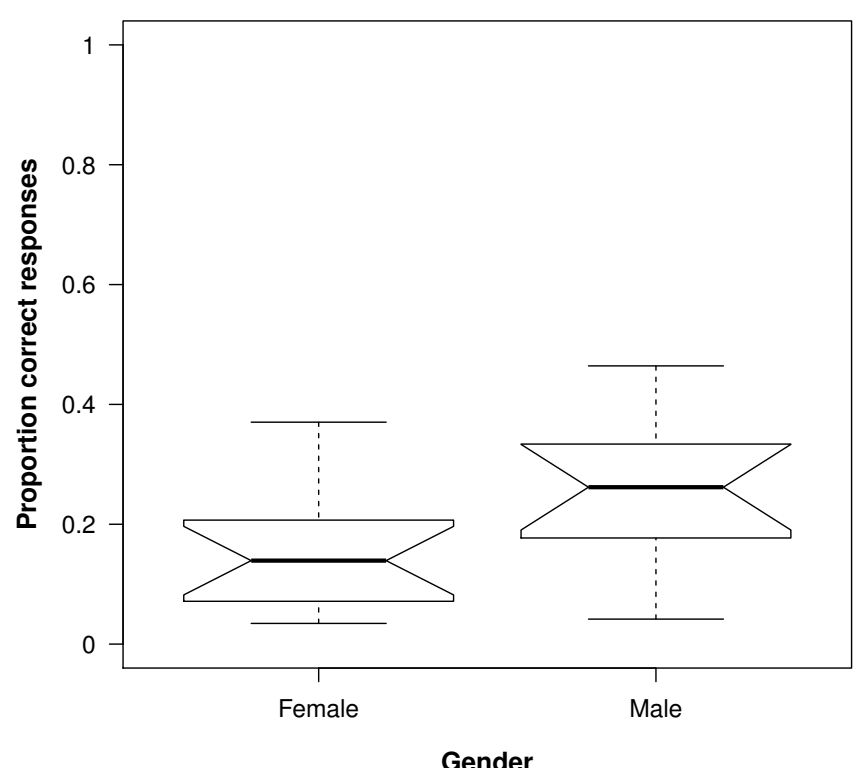

Figure 2. The figure shows the proportion of correct antisaccades by gender.

gender and oculomotor control to the regression model (see further discussion below).

\section{Saliency features in online adverts}

The results reported in this section contain some descriptive statistics of levels of visual saliency found in online adverts. These saliency levels were computed from the web page screen recordings, and are shown in Table 1 below. The total number of unique ads found in the screen recordings were 396, which means that each child was "potentially exposed" to about 15 ads on average during the course of the 7 minute surfing sessions (Gidlöf et al., 2012). The average presentation time of adverts was 20 seconds, and the average size of an advert was around $350 \times 350$ pixels (ca $7 \%$ of the screen size).

\section{Visual attention to online adverts}

We hypothesized that children's visual attention to internet adverts could be described as a function of saliency variables (presentation time, motion, luminance, edges, and size of adverts), combined with individual factors (gender, oculomotor control, and internet use). Since we found significant differences between boys and girls on the anti-saccade test, we also thought that including an interaction between gender and oculomotor control would contribute to the explanatory power of the model. To test this hypothesis, a mixed-effects multiple regression analysis was performed, in which participants were treated as a random factor. Tests for multicollinearity indicated a low level
Holmberg, N., Holmqvist, K., \& Sandberg, H. (2015) Online Adverts Affect Children's Attention

Table 1

Descriptive statistics of advert saliency measures.

\begin{tabular}{lcc}
\hline Saliency measure & Mean & SD \\
\hline Duration (sec) $^{1}$ & 20.47 & 45.45 \\
Rel. Size $^{2}$ & 0.072 & 0.056 \\
Rel. Motion $^{3}$ & 0.180 & 0.220 \\
Rel. Edges $^{3}$ & 0.085 & 0.052 \\
Rel. Luminance $^{3}$ & 0.068 & 0.065 \\
\hline
\end{tabular}

1. Durations were added when adverts were visible on the web page. Time when adverts were completely invisible due to page scrolling is not included.

2. Size values were calculated for each video frame and averaged over each unique advert. Average size presented as a proportion relative to the display size at a resolution of 1680 $\times 1050$ pixels.

3. Saliency values were calculated for each video frame as a proportion of pixels relative to the saliency value of the entire web page. Relative saliency values were then averaged over each unique advert.

interdependence between predictors (VIF=1.63 for gender, 1.16 for oculomotor control, 1.47 for internet usage time, 1.29 for ad presentation time, 2.23 for ad edges, 2.64 for ad luminance, 1.91 for ad motion, and 2.69 for ad size). Also, multiple tests for outlier cases were performed. Results of the regression analysis provided partial confirmation for the research hypotheses. The order, coefficients and p-values for the predictors are given in Table 2 below.

Table 2

Effects of advert saliency and individual factors on visual attention.

\begin{tabular}{lllll}
\hline & Coeff. & $\begin{array}{l}\text { Std. } \\
\text { Error }\end{array}$ & $\begin{array}{l}\mathrm{z} \\
\text { value }\end{array}$ & $\begin{array}{l}\mathrm{P}= \\
\mathrm{r}(>|\mathrm{z}|)\end{array}$ \\
\hline (Intercept) & 4.04 & 1.02 & 3.95 & 0.0001 \\
Adv. Dur. & 0.86 & 0.13 & 6.50 & 0.0000 \\
Adv. Motion & 1.01 & 0.13 & 7.46 & 0.0000 \\
Adv. Edges & 0.68 & 0.29 & 2.34 & 0.0191 \\
Adv. Lum. & -0.61 & 0.23 & -2.72 & 0.0066 \\
Adv. Size & 0.00 & 0.00 & 0.98 & 0.3249 \\
Sub. Int.Use & -0.07 & 0.17 & -0.43 & 0.6695 \\
Sub. Gen. (m) & -1.18 & 0.77 & -1.53 & 0.1256 \\
Sub. Gaze & -4.47 & 1.63 & -2.74 & 0.0061 \\
Gen. $\times$ Gaze & 3.48 & 2.73 & 1.27 & 0.2035 \\
\hline
\end{tabular}

Predictors of children's visual attention to internet adverts. Advert saliency measures (Adv.) were log-transformed before entered into the regression model. Measures of individual differences (Sub.) include internet use (Int.Use), gender (Gen.), and gaze control (Gaze). Interaction between gender and gaze control is given last. 
Journal of Eye Movement Research $8(2): 2,1-10$

The deviance of the described regression model was $371.28(\mathrm{df}=386)$, which proved to be considerably lower than the deviance of the corresponding unconditional null model in which all predictors were discarded (deviance=542.12, $\mathrm{df}=395$ ). A chi-square test showed that the difference in deviance between these models was significant at the highest level $(\mathrm{p} \leq 0.001)$, providing evidence that the selected group of predictors contributed significantly to explaining the observed variance in the dependent variable. Excluding the nonsignificant variables, thus providing a reduced model, did not significantly improve the deviance, and therefore a reduced model was rejected. Based on this evidence, we conclude that the best fitting model for predicting children's visual attention to internet adverts is a linear combination of the variable set given in Table 2 above.

\section{Discussion}

In relation to the hypotheses presented at the outset of this study, the main findings are as follows: (H1) low-level visual saliency features of internet adverts have a strong influence in determining children's visual attention; (H2) children's individual level of oculomotor control seems to modulate the effect of visual saliency features in internet ads, and thus oculomotor control impacts on children's visual exposure to advertising; (H3) gender does not seem to directly influence children's attention to internet adverts; $(\mathbf{H} 4)$ children's individual level of weekly internet usage time does not influence their attention to adverts. Before discussing these findings separately below, it is also useful to recapitulate the measure of visual attention utilized in this study. Visual attention has been operationalized as binary variable expressing if children looked at an internet ad (as measured by eye-tracking equipment). Thus, this dependent measure only takes into account if an advert was looked at, and not how much adverts were attended. The reason behind this operationalization was our assumption that this dependent measure is more sensitive to the low-level saliency features investigated in this study.

\section{Advert saliency and visual attention}

Several of the measures of visual saliency employed in this study proved to have a significant effect on children's visual attention to internet adverts. As predictors, these saliency features typically have positive relations to the participants' visual attention to ads, meaning that the higher saliency value, the more visual attention. The only temporal measure included in the analysis was advert presentation time, which had a high positive coefficient. Arguably, this effect is not due to visual saliency so much as to the increased probability of a participant looking at an ad the longer it is presented. In the spatial domain, motion turned out to be the saliency feature with the strongest impact on
Holmberg, N., Holmqvist, K., \& Sandberg, H. (2015) Online Adverts Affect Children's Attention

visual attention. Motion was defined as the sum of different pixels between two successive frame pairs, averaged for each advertisement. This definition captures several types of motion, e.g. abrupt onset, flicker, and object motion (Franconeri \& Simons, 2003). It does not, however, take into account the speed of motion, which might also be and important aspect of motion saliency.

Similar to motion, edge information also had a positive coefficient in the regression analysis, meaning that more edges in an online advert (i.e. more spatial clutter) increased the probability of children fixating this advert. The effect of edge information was considerably weaker than that of motion. Advert luminance had a significantly negative regression line, implying that as relative luminance values decreased, visual attention increased. A reasonable interpretation of this negative association would be that when ads consisted of dark patches on a light web page (low relative luminance), this drew more attention than when adverts consisted of light patches on a dark web page (high relative luminance). As a bottom-up saliency feature, relative luminance in internet adverts ranked in between motion (high impact) and edges (low impact).

Interestingly, advert size was the only saliency feature in the analysis that did not have a detectable effect on the participants' vision. This runs counter to many previous studies, which often report a positive association between more visual attention and larger ads (Gidlöf et al., 2012). The exact reason for this deviating result is difficult to state, but in the case of online advertising, it is possible that some previous studies may have confounded the advert size factor with for example motion and/or luminance, by not using real-time screen recordings of participants' web interaction, but rather use static screen dumps of each web page. If this is the case, it points to the benefits of using the methodology utilized in this study when analyzing web stimuli. Looking at all the saliency measures together, they appear to represent a "hierarchy of saliency effects", with motion at the strong end of the spectrum and edge information at the weak end.

A different type of advert saliency which was not addressed in the current study concerns audio in online advertising. On many video websites, e.g. youtube.com (which proved to be very popular among participants in this study), virtually all advertising is presented as videos with integrated audio. This audio signal ranges from speech, which could conceivably guide allocation of visual attention within the advertising video, to music, which could engage the viewer emotionally. Although the effects of audio on visual attention have been studied under the audiovisual integration paradigm (Koelewijn, Bronkhorst, \& Theeuwes, 2010), it is unlikely that audio as a separate factor would contribute to explaining which adverts were visually attended in the current study. 
Journal of Eye Movement Research $8(2): 2,1-10$

\section{Gaze control and advert attention}

Among the individual factors investigated in this study, oculomotor control emerged as one measure that actually impacts children's visual attention to adverts. The regression analysis suggested a strong negative coefficient for this predictor, meaning that better success rates in the anti-saccade test were associated with significantly fewer gazes to internet adverts. Or, the more voluntary control children had to inhibit their reflexive eye movements, the less they tended to look at advertising. Interestingly, the very fact that oculomotor control had an effect on these children's visual behavior suggests that the online adverts they encountered actually contained a considerable amount of low-level visual saliency. If the online adverts on these websites had not impinged on the children's visual system, high or low gaze control would probably not have been associated with the amount of visual attention to the adverts.

The results on the anti-saccade test showed that the average success rate was around $21 \%$ in the sample as a whole, which is considerably lower than what has been observed in adult populations. Success rates around $80 \%$ have been reported for adults in recent large-scale studies (Hutton \& Ettinger, 2006). Conversely, saccade latencies in the child sample were higher than what would typically be expected in an adult population. On the other hand, our results on gaze control were very similar to other research findings on saccadic eye movements in children (Bucci \& Seassau, 2012; Fukushima et al., 2000). However, there are studies reporting a somewhat higher success rate for 9-10 year old children in the anti-saccade paradigm (Kramer et al., 2005; Munoz \& Everling, 2004). These differences could simply reflect that the anti-saccade stimuli material have been constructed differently across studies. One question these previous studies do not address is whether there are differences in success rate between boys and girls. In the current study, the difference in oculomotor control depending on gender turned out to be statistically significant, which calls for further investigation. One possibility is that the boys' higher success rate is linked to their engagement in particular types of extracurricular activities that require high levels of visual focus, e.g. video gaming and/or sports. The interaction between gender and oculomotor control did not contribute significantly to the regression model.

\section{Individual factors and advert attention}

Gender did not prove to have a reliable effect on visual attention to internet adverts in this study. This result probably reflects a general lack of evidence for strong gender effects in the larger field of vision/cognitive research. A large meta-study concluded that supposedly "well-established" gender differences were actually rather weak across a number of cognitive domains and processes (Hyde, 1981). Also,
Holmberg, N., Holmqvist, K., \& Sandberg, H. (2015) Online Adverts Affect Children's Attention

large survey studies on children's internet usage patterns such as (NORDICOM-Sweden, 2011), indicate that boys tend to visit online gaming sites whereas girls are more likely to engage in social media sites. This pattern was not replicated in the current sample, in which both boys and girls listed online gaming sites as their most preferred internet content. This might explain the similarities in advertising exposure between genders. Furthermore, gender differences in attention to internet adverts might not be that pronounced within the particular age group investigated in this study.

Perhaps more surprisingly, weekly internet usage time also did not contribute to predicting children's visual attention to internet adverts. We reasoned that the amount of time a child spends on using internet daily/weekly would roughly approximate the concept of internet "expertise" (or internet literacy). Such expertise is known to have a powerful altering effect on visual behavior in many professional areas, effectively distinguishing experts and novices (Jarodzka et al., 2010). Based on this research, we expected that children who spent more time on the internet would exhibit a different visual behavior towards internet ads as compared to their peers, e.g. avoiding more ads. The reason internet usage time did not contribute as a predictor of advertising exposure in this study might have to do with the free, unconstrained nature of the web browsing task. Since expertise is generally directed towards solving a specific task, the effect of internet usage time could have been more pronounced if we had given the children e.g. an information search task, in which visual attention to internet adverts would conflict with effectively solving the specified task.

\section{Conclusions}

The results of this study provide a chain of evidence and explanations as to why some children report that they experience internet advertising as disturbing and sometimes even coercing in its calls for visual attention (Martinez, Sandberg, \& Jarlbro, 2012). An initial antisaccade test showed that children in 3rd grade (aged 9-10 years) generally have difficulties to inhibit reflexive eye movements towards abrupt onset of peripheral distractors. Saliency analyses of the web pages that children typically visit, revealed that the adverts children are exposed to during free internet surfing contain substantial amounts of motion relative to the surrounding web page. These results were then fed into a multiple regression analysis that showed clear evidence that motion and luminance in internet adverts indeed are powerful predictors of attracting children's visual attention to ads. But perhaps more importantly, the regression analysis showed that children's individual scores on the anti-saccade task modulates their sensitivity to low-level saliency in internet adverts. Although children in this age group are sensitive to lowlevel saliency, some children may be more at risk of 
Journal of Eye Movement Research $8(2): 2,1-10$

having their goal-directed surfing activities disrupted by adverts. This raises questions about how advertising on children's websites could be redesigned in order to give children equal opportunities on the web (Livingstone \& Haddon, 2009).

A wider objective of the current study is to give publicly funded research a possibility to catch up with the commercially restricted knowledge about saliency factors that drive visual attention that is already generated within advertising companies. Given the the business model of the internet, it seems unlikely that online advertising content could be effectively regulated, but knowledge about the relationship between visual saliency and attention could help researchers and developers to build better browsers that can filter out distracting advertising content. This knowledge can also be used by teachers in order to educate children about visual saliency in online advertising and how it affects visual attention, and thus raise children's awareness concerning attentional effects of commercial content.

Intriguing questions that remain to be answered concerns the effect of higher-level content categories in internet adverts, for example celebrities, animated cartoons and animals. It seems likely that there could be an interaction between low-level saliency features and high-level saliency, so that e.g. a combination of motion and celebrities would cause even more visual attention in children. Additionally, it is urgent to find out what kind of visual and semantic information children encode while attending to internet advertising. This could be tested with a recognition and recall test on internet adverts immediately after the surfing session. The focus on free internet browsing and the effects of low-level saliency features in current study should be understood as part of a larger project that also intends to investigate task-oriented browsing and the effects of behaviorally targeted and relevant online advertising.

\section{Acknowledgements}

This research was supported by the Crafoord Foundation (grant id 20100899) and the Swedish Research Council (grant id 421-2010-1982). There are no commercial interests or relationships involved in this research. Correspondence concerning this article should be addressed to: Nils Holmberg, Dept. of Communication and Media, Lund University, PO Box 201, 22100 Lund, Sweden. Email: nils.holmberg@kom.lu.se

\section{References}

Ahlström, C., Nyström, M., Holmqvist, K., Fors, C., Sandberg, D., Anund, A., ... Akerstedt, T. (2013). Fit-forduty test for estimation of drivers' sleepiness level: Eye movements improve the sleep/wake predictor. Transportation Research Part C: Emerging Technologies, 26(0), 20 - 32. Retrieved from http://www.sciencedirect.com/ science/article/pii/S0968090X1200099x doi: http:// dx.doi.org/10.1016/j.trc.2012.07.008
Holmberg, N., Holmqvist, K., \& Sandberg, H. (2015) Online Adverts Affect Children's Attention

Antoniades, C., Ettinger, U., Gaymard, B., Gilchrist, I., KristjÃąnsson, A., Kennard, C., ... Carpenter, R. (2013). An internationally standardised antisaccade protocol. Vision Research, 84(0), 1 - $5 . \quad$ Retrieved from http://www.sciencedirect.com/science/article/ pii/S0042698913000357 doi: http://dx.doi.org/10.1016/ j.visres.2013.02.007

Bradski, G. (2000). Open computer vision. Dr. Dobb's Journal of Software Tools.

Bucci, M., \& Seassau, M. (2012). Saccadic eye movements in children: a developmental study. Experimental Brain Research, 1-10. Retrieved from http://dx.doi.org/10.1007/ s00221-012-3192-7 (10.1007/s00221-012-3192-7)

Carlsson, U. (2012). Barn och ungas medieanvändning $i$ nätverkssamhället. Göteborg: Nordicom.

Council, T. S. M. (2010). Ungar E medier 2010 [elektronisk resurs] : fakta om barns och ungas användning och upplevelser av medier. Stockholm: Medierådet.

Davidson, M. C., Amso, D., Anderson, L. C., \& Diamond, A. (2006). Development of cognitive control and executive functions from 4 to 13 years: Evidence from manipulations of memory, inhibition, and task switching. Neuropsychologia, 44(11), 2037-2078.

Eenshuistra, R. M., Ridderinkhof, K. R., \& Molen, M. W. (2004). Age-related changes in antisaccade task performance: Inhibitory control or working-memory engagement? Brain and Cognition, 56(2), 177-188.

Engbert, R., \& Kliegl, R. (2003). Microsaccades uncover the orientation of covert attention. Vision Research, 43(9), 1035 - 1045. Retrieved from http://www.sciencedirect.com/ science/article/pii/S0042698903000841 doi: http:// dx.doi.org/10.1016/S0042-6989(03)00084-1

Foulsham, T., \& Underwood, G. (2008). What can saliency models predict about eye movements? spatial and sequential aspects of fixations during encoding and recognition. Journal of Vision, 8(2). Retrieved from http://wwW . journalofvision.org/content/8/2/6. abstract doi: 10 $.1167 / 8.2 .6$

Franconeri, S., \& Simons, D. (2003). Moving and looming stimuli capture attention. Attention, Perception, \& Psychophysics, 65, 999-1010. Retrieved from http://dx.doi .org/10.3758/BF03194829 (10.3758/BF03194829)

Fukushima, J., Hatta, T., \& Fukushima, K. (2000). Development of voluntary control of saccadic eye movements: I. age-related changes in normal children. Brain and Development, 22(3), $173-180$.

Gidlöf, K., Holmberg, N., \& Sandberg, H. (2012). The use of eye-tracking and retrospective interviews to study teenagers' exposure to online advertising. Visual Communication, 11(3), 329-345. Retrieved from http://vcj . sagepub.com/content/11/3/329. abstract doi: 10 $.1177 / 1470357212446412$

Goldfarb, A., \& Tucker, C. E. (2011). Online advertising, behavioral targeting, and privacy. Communications of the ACM, 54(5), 25-27.

Holmberg, N., Sandberg, H., \& Holmqvist, K. (2014). Advert saliency distracts children's visual attention during task-oriented internet use. Frontiers in Psychology, 5, 51. Retrieved from http://www. frontiersin.org/ cognition/10.3389/fpsyg.2014.00051/abstract doi: 10 $.3389 /$ fpsyg.2014.00051

Hutton, S. B., \& Ettinger, U. (2006). The antisaccade task as a research tool in psychopathology: a critical review. Psy- 
Journal of Eye Movement Research

$8(2): 2,1-10$

chophysiology, 43(3), 302-313.

Hyde, J. S. (1981). How large are cognitive gender differences?: A meta-analysis. American Psychologist, 36(8), 892901.

Itti, L., \& Koch, C. (2000). A saliency-based search mechanism for overt and covert shifts of visual attention. Vision Research, 40(10âĂŞ12), 1489 - 1506. Retrieved from http://www.sciencedirect.com/science/article/ pii/S0042698999001637 doi: 10.1016/S0042-6989(99) 00163-7

Jarodzka, H., Scheiter, K., Gerjets, P., \& van Gog, T. (2010). In the eyes of the beholder: How experts and novices interpret dynamic stimuli. Learning and Instruction, 20(2), 146 - 154. Retrieved from http://www.sciencedirect.com/ science/article/pii/S0959475209000255 (<ce:title>Eye tracking as a tool to study and enhance multimedia learning</ce:title>) doi: 10.1016/j.learninstruc.2009.02.019

Klein, C., \& Foerster, F. (2001). Development of prosaccade and antisaccade task performance in participants aged 6 to 26 years. Psychophysiology, 38(2), 179-189. Retrieved from http://dx.doi.org/10.1111/1469-8986.3820179 doi: 10 .1111/1469-8986.3820179

Koelewijn, T., Bronkhorst, A., \& Theeuwes, J. (2010). Attention and the multiple stages of multisensory integration: A review of audiovisual studies. Acta psychologica, 134(3), 372-384.

Kramer, A. F., Gonzalez de Sather, J. C. M., \& Cassavaugh, N. D. (2005). Development of attentional and oculomotor control. Developmental Psychology, 41(5), 760-772. doi: 10.1037/0012-1649.41.5.760

Livingstone, S. M., \& Haddon, L. (2009). Young people in the european digital media landscape : a statistical overview with an introduction. Göteborg: NORDICOM.

Ludwig, C. J. H., Ranson, A., \& Gilchrist, I. D. (2008). Oculomotor capture by transient events: A comparison of abrupt onsets, offsets, motion, and flicker. Journal of Vision, 8(14). Retrieved from http://www . journalofvision.org/ content/8/14/11.abstract doi: 10.1167/8.14.11

Malcolm, G. L., \& Henderson, J. M. (2010). Combining top-down processes to guide eye movements during realworld scene search. Journal of Vision, 10(2).

Martinez, C., Sandberg, H., \& Jarlbro, G. (2012). Children's uses of, attitudes towards, and practices in relation to, online advertising. an interview study with swedish 9 year
Holmberg, N., Holmqvist, K., \& Sandberg, H. (2015) Online Adverts Affect Children's Attention

line advertising. an interview study with swedish 9 year olds. Manuscript in preparation.

Martinez, C., Sandberg, H., \& Jarlbro, G. (2013). Children's views and practices regarding online advertising. an interview study with swedish nine-year-olds. Nordicom Review, 34(2), 107-121.

Medierådet. (2013). Ungar E medier 2013. Stockholm: Author.

Munoz, D. P., \& Everling, S. (2004, Mar). Look away: the antisaccade task and the voluntary control of eye movement. Nat Rev Neurosci, 5(3), 218-228. Retrieved from http:// dx.doi.org/10.1038/nrn1345 doi: 10.1038/nrn1345

Nordicom. (2013). Nordicom-sveriges mediebarometer. 2012. Gothenburg: Gothenburg: NORDICOM, Gothenburg University.

NORDICOM-Sweden. (2011). Nordicom-sveriges mediebarometer. 2010 (Vol. 1). Göteborg: Nordicom-Sverige, Nordiskt informationscenter för medie- och kommunikationsforskning, Göteborgs universitet.

Parkhurst, D. J., \& Niebur, E. (2004). Texture contrast attracts overt visual attention in natural scenes. European Journal of Neuroscience, 19(3), 783-789.

Patterson, C. (2008). Child development. Boston: McGraw-Hill Higher Education.

Sandberg, H., Gidlöf, K., \& Holmberg, N. (2011, Jan). Children's exposure to and perceptions of online advertising. International Journal of Communication; Vol 5 (2011). Retrieved from http://ijoc.org/ojs/index.php/ ijoc/article/view/716/502

Singer, D. G., \& Singer, J. L. (2011). Handbook of children and the media (2. ed. ed.). London: SAGE.

Sundin, S. (2013). Den svenska mediemarknaden 2013. Göteborg: Nordicom.

Tatler, B. W., Hayhoe, M. M., Land, M. F., \& Ballard, D. H. (2011). Eye guidance in natural vision: Reinterpreting salience. Journal of vision, 11(5).

Zanelli, J., Simon, H., Rabe-Hesketh, S., Walshe, M., McDonald, C., Murray, R. M., \& MacCabe, J. H. (2005). Eye tracking in schizophrenia: Does the antisaccade task measure anything that the smooth pursuit task does not? Psychiatry Research, 136(2âĂŞ3), 181 - 188. Retrieved from http://www.sciencedirect.com/science/article/ pii/S0165178105001514 doi: http://dx.doi.org/10.1016/ j.psychres.2004.12.008 